\title{
Neuromarkers from Whole-Brain Functional Connectivity Reveal the Cognitive Recovery Scheme for Overt Hepatic Encephalopathy after Liver Transplantation
}

\author{
Yue Cheng, ${ }^{1}$ Wen Shen, ${ }^{1}$ Junhai Xu, ${ }^{2}$ Rachel C. Amey, ${ }^{3}$ Li-Xiang Huang, ${ }^{1}$ Xiao-Dong Zhang, ${ }^{1}$ Jing-Li Li, ${ }^{1}$ \\ Cameron Akhavan, ${ }^{5}$ Ben A. Duffy, ${ }^{5}$ Julia Pia Simon, ${ }^{5}$ Wenjuan Jiang, ${ }^{4}$ Mengting Liu, ${ }^{5}$ and Hosung Kim ${ }^{5}$
}

\author{
https://doi.org/10.1523/ENEURO.0114-21.2021
}

\begin{abstract}
${ }^{1}$ Department of Radiology, Tianjin First Center Hospital, Tianjin 300192, People's Republic of China, ${ }^{2}$ Tianjin Key Laboratory of Cognitive Computing and Application, School of Artificial Intelligence, College of Intelligence and Computing, Tianjin University, Tianjin 300350, People's Republic of China, ${ }^{3}$ U.S. Army Research Institute for the Behavioral and Social Sciences, Fort Belvoir, Virginia 22060-5610, ${ }^{4}$ College of Pharmacy, Western University of Health Sciences, Pomona, California 91766-1854, and 5 USC Mark and Mary Stevens Neuroimaging and Informatics Institute, Keck School of Medicine of USC, University of Southern California, Los Angeles, California 90033
\end{abstract}

\begin{abstract}
Neurocognitive impairment is present in cirrhosis and may be more severe in cirrhosis with overt hepatic encephalopathy $(\mathrm{OHE})$. Liver transplantation (LT) can restore liver function, but how it reverses the impaired brain function is still unclear. MRI of resting-state functional connectivity can help reveal the underlying mechanisms that lead to these cognitive deficits and cognitive recovery. In this study, 64 patients with cirrhosis $(28$ with $\mathrm{OHE} ; 36$ without $\mathrm{OHE}$ ) and 32 healthy control subjects were recruited for resting-state fMRI. The patients were scanned before and after LT. We evaluated presurgical and postsurgical neurocognitive performance in cirrhosis patients using psychomotor tests. Network-based statistics found significant disrupted connectivity in both groups of cirrhotic patients, with OHE and without OHE, compared with control subjects. However, the presurgical connectivity disruption in patients with OHE affected a greater number of connections than those without OHE. The decrease in functional connectivity for both OHE and non-OHE patient groups was reversed after LT to the level of control subjects. An additional hyperconnected network (i.e., higher connected than control subjects) was observed in OHE patients after LT. Regarding the neural-behavior relationship, the functional network that predicted cognitive performance in healthy individuals showed no correlation in presurgical cirrhotic patients. The impaired neural-behavior relationship was re-established after LT for non-OHE patients, but not for OHE patients. OHE patients displayed abnormal hyperconnectivity and a persistently impaired neural-behavior relationship after
\end{abstract}

\section{Significance Statement}

After liver transplantation, brain functional impairment induced by cirrhosis shows some recovery in terms of both brain functional connectivity and cognitive task performance. However, cirrhotic patients with and without OHE present different recovery paths. We show that a hyperconnected network emerges for OHE patients only after liver transplantation, within which the connectivity is abnormally higher than that in healthy control subjects. Furthermore, the predictive power of the neuromarkers for cognitive task performance returns to normal in non-OHE patients only, but not in OHE patients. These findings suggest that OHE patients may exhibit functional reorganization in brain functional MRI after liver transplantation. That is, the brain connectivity responsible for cognitive task performance alters, and the brain-behavior relationship is reshaped. 
LT. Our results suggest that patients with OHE may undergo a different trajectory of postsurgical neurofunctional recovery compared with those without, which needs further clarification in future studies.

Key words: connectome-behavior predictive mapping; functional network; liver transplantation; neuropsychological assessment; overt hepatic encephalopathy

\section{Introduction}

Overt hepatic encephalopathy (OHE) is one of the most prominent complications in cirrhotic patients. It decreases a patient's quality of life, in addition to lowering the survival rate. Neurocognitive impairment is present in cirrhosis and occurs along a continuous spectrum. These impairments are accelerated by episodes of OHE (Bajaj et al., 2010), which can often irreversibly impair attention, learning ability, and executive function (Campagna et al., 2014). Studies have demonstrated (Bajaj et al., 2010; Umapathy et al., 2014) poorer neurocognitive performance in patients with OHE than in those without.

Liver transplantation (LT) is the only effective treatment for end-stage cirrhosis (Cárdenas and Ginès, 2011). Successful LT can restore liver function completely. However, to what extent cognitive dysfunction can be recovered, and to what extent OHE can impact the recovery process, is still unclear (Sotil et al., 2009; Ahluwalia et al., 2016; Cheng et al., 2017).

Neurocognitive deficits and recovery are usually assessed by extensive psychometric neurocognitive testing for cirrhosis studies. Previous studies (Bajaj et al., 2009; Sotil et al., 2009; Campagna et al., 2014) consistently demonstrate that patients with cirrhosis, both with and without OHE, produce low performance in the MiniMental State Exam, the Number Connection Test (NCT), and the Digit Symbol Test (DST). This suggests that the impairment led by cirrhosis is not limited to a specific functional domain, but rather includes a wide range of cognitive functions including short-term memory (recall), attention, language, comprehension, motor skill, as well as executive functions. These impairments were reflected by brain atrophy and decreased functional connectivity (FC; Chen et al., 2012). Functional MRI, particularly resting-state functional connectivity (Qi et al., 2012, 2014; Chen et al., 2016; Cheng et al., 2017; Zhang et al., 2017a, b), could help to further unravel the underlying

Received March 18, 2021; accepted July 26, 2021; First published August 10, 2021.

The authors declare no competing financial interests.

Author contributions: Y.C., M.L., and H.K. designed research; Y.C. and M.L. performed research; W.S., J.X., R.C.A., L.-X.H., X.-D.Z., J.-L.L., and W.J. contributed unpublished reagents/analytic tools; J.X. and M.L. analyzed data; R.C.A., C.A., B.A.D., J.P.S., W.J., M.L., and H.K. wrote the paper.

This study has received funding from the National Natural Science Foundation of China (Grants 81601482, 61703302, and 81801776). The views expressed in this article are those of the authors and do not reflect the official policy or position of the Department of the Army, Department of Defense, or the U.S. Government.

Correspondence should be addressed to Mengting Liu at mliu@ini.usc.edu. https://doi.org/10.1523/ENEURO.0114-21.2021

Copyright (C) 2021 Cheng et al.

This is an open-access article distributed under the terms of the Creative Commons Attribution 4.0 International license, which permits unrestricted use, distribution and reproduction in any medium provided that the original work is properly attributed. mechanisms that lead to these cognitive deficits and recovery. Several studies have investigated the association between impaired cognitive functions and the functional connectivity related to these functions using hypothesisdriven approaches (Qi et al., 2012; García-García et al., 2018). However, which localized functional connectivity corresponds to which cognitive functions is still not well established (Poldrack, 2010; Barch et al., 2013; Poldrack and Yarkoni, 2016). Furthermore, studies of patients with various brain injuries have shown evidence that functional hyperconnectivity or brain reorganization may occur in response to the initial brain injury, leading to broader connectivity changes beyond the changes in the relevant brain regions (Castellanos et al., 2010; Grefkes and Ward, 2014; Bharath et al., 2015; Bernier et al., 2017). This makes it difficult for investigators to arrive at a clear conclusion for their neuroimaging data when applying hypothesis-driven approaches, where only hypothetically relevant functional regions are investigated. Hence, searching for possible biomarkers across the whole brain network, via data-driven approaches, without restriction within known brain functional regions, connections, and functional networks, is critical to understanding the cirrhosis-associated neurocognitive impairments and recovery.

Associated brain biomarkers can often make inferring behavior from functional connectivity difficult (Woo et al., 2017). The biomarkers are typically found using a hypothesis driven approach, based on findings from previous related studies (Yarkoni et al., 2011). To circumvent these potential problems, further analyses need to be conducted that require a technique to understand intrinsic network properties, an approach that is capable of isolating unique patterns characterizing cognitive performance.

In this study, we used a data-driven approach that combined a cognitive task with whole-brain FC to provide a new perspective on cognitive deficits and recovery for $\mathrm{OHE}$ and non-OHE cirrhotic patients before and after transplant surgery. We evaluated neurocognitive deficits in cirrhotic patients and recovery after LT using a standard psychomotor cognitive task that is reliant on a variety of functional neural components like sensorimotor, attention, memory, and executive functions (Miyake et al., 2000). To avoid limiting our study to the analysis of brain regions, connections, and functional networks hypothetically related to neurocognitive impairment in patients with cirrhosis, we used "connectome predictive mapping" (CPM) to identify unique patterns characterizing cognitive performance. The CPM searches for all possible pairs of brain regions and their associated connectivity values, consequently constructing a connectome model that optimizes the association with behavioral scores (Finn et al., 2015; Rosenberg et al., 2017; Shen et al., 2017). The predictive power and the 


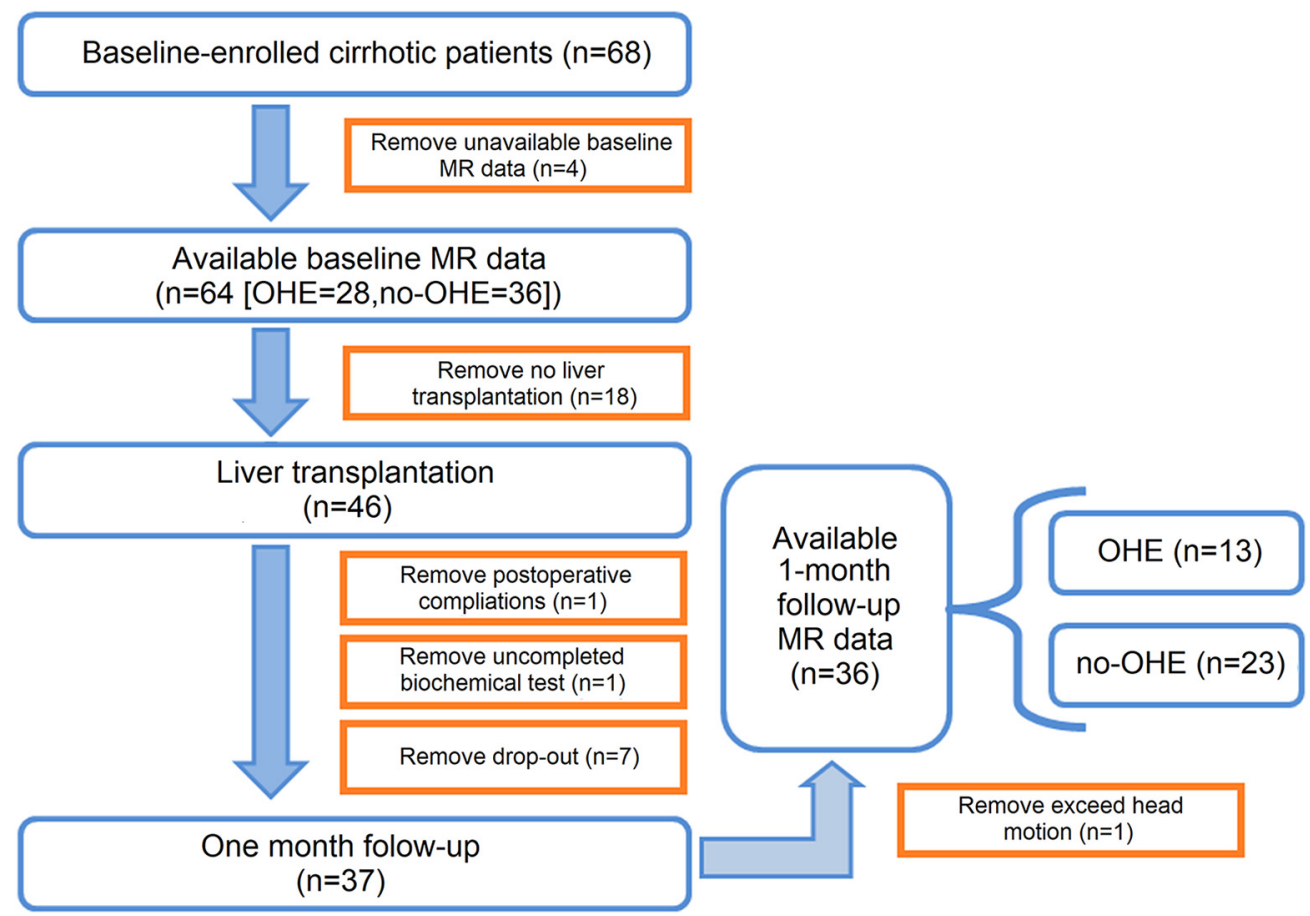

Figure 1. Subjects involved in this study. Sixty-eight cirrhotic patients were enrolled in this study, and a subset of patients was removed from the subject pool for the following reasons: unavailable baseline MR data, not undergoing LT, postoperative complications, incomplete biomedical examination, dropping out, or having excess head movement (see panels for exact numbers). In total, 28 patients with OHE and 36 without OHE were analyzed before LT; 13 patients with a history of OHE and 23 patients without a history of OHE participated in the study after the LT operation.

robustness of the connectome was further validated using cross-validation on novel/unseen subjects. As such, CPM is a data-driven prediction method that can identify what aspects of network properties characterize cognitive task performance in individuals. Using CPM, we address questions such as the following. (1) Compared with healthy control subjects ( $\mathrm{HCs})$, are the disrupted or altered functional connections in cirrhotic patients with $\mathrm{OHE}$, or in both cirrhotic groups regardless of OHE? (2) Are these connections recovered, reversed, or reorganized after LT treatment? (3) Are the re-established connections after LT the same as for control subjects or are they different from control subjects because of brain reorganization? (4) Is the normal relationship that is observed between a healthy brain and cognitive performance impaired in presurgical cirrhotic patients, and is this recovered after LT?

\section{Materials and Methods}

\section{Subjects}

In this prospective study, approval was obtained from the Ethics Committee of institutional review board from Tianjin First Center Hospital. All subjects provided written informed consent before being included in the study. From November 2013 to January 2018, 64 patients with end-stage cirrhosis (28 patients with a history of OHE episodes; 36 patients without a history of OHE episodes) scheduled to undergo LT were recruited from the Department of Transplantation Surgery in Tianjin First Center Hospital. Thirty-two of the 64 patients have been previously reported (Cheng et al., 2015; Zhang et al., 2017b) investigating regional functional activity, whereas in this study we evaluated functional connectivity and specifically its predictive power for behavior (i.e., the psychomotor cognitive performance score). LT candidates who completed all necessary laboratory examinations, neuropsychological tests, and baseline MRI were included. Participants were excluded if they reported the following: (1) a history of drug or alcohol abuse; (2) the presence of any noticeable brain lesions on conventional MR, such as a tumor or stroke; (3) any major neurologic or psychiatric disorders; (4) history of liver cancer; (5) previous liver or other organ transplantation; and (6) head motion of $1.5 \mathrm{~mm}$ or $1.5^{\circ}$ during MRI. The etiology of the cirrhotic patients included type B hepatitis $(n=35)$, type $C$ hepatitis $(n=15)$, primary biliary cirrhosis $(n=8)$, and cryptogenic cirrhosis $(n=6)$. Thirty-six of these patients eventually received successful LT and completed the 1month follow-up examination. Included patients had no complications such as acute transplant rejection, liver failure, severe biliary complications, or any neurologic complications, such as alterations in mental status, seizures, and focal motor deficits. A detailed flowchart of this study is provided in Figure 1.

Thirty-two age- and sex-matched HCs were recruited from the local community. All HCs had no diseases or history of liver or neurologic diseases. All HCs were selfidentified as right handed, with normal sight, and had completed neuropsychological tests (see details in Neuropsychological tests). 


\section{Laboratory examinations}

All patients completed blood laboratory tests to evaluate liver function 1 week before their MRI scan, both before and 1 month after LT. These tests included prothrombin time, albumin, and total bilirubin. For the preoperative patients, their Child-Pugh score was used to assess liver dysfunction. Venous blood ammonia was also tested for cirrhotic patients. No blood laboratory tests were performed for HCs.

\section{Neuropsychological tests}

All patients and HCs underwent two typical neuropsychological tests to evaluate cognitive function before the MRI scan. These tests included the NCT type A (NCT-A; abbreviated as NCT in all text below) and the DST (Weissenborn et al., 2001). The NCT and DST are part of the Psychometric Hepatic Encephalopathy Score (Ferenci et al., 2002; Weissenborn et al., 2005) and are ideal for this study as they are supported by a variety of functional neural components given their reliance on different sensorimotor, attention, memory, and executive functions (Miyake et al., 2000). The DST and NCT have undergone repeated and rigorous psychometric validation such as test-retest reliability and discriminant validity in a range of patient samples (Matarazzo and Herman, 1984; Jaeger, 2018; Zeng et al., 2020). In the NCT task, participants were required to connect randomly placed figures in order as quickly as possible to measure psychomotor speed and attention (Bajaj et al., 2009). Completion time is indicative of performance, and worse performance is indicated by a longer completion time. The DST is a measure of complex visuomotor tracking and learning, which emphasizes sustained attention, response speed, and visuomotor integration (Bajaj et al., 2011). Digits from one to nine and corresponding symbols are displayed in front of the subjects, who are then asked to fill in the blanks with the symbol that matched each digit. Participants have $90 \mathrm{~s}$ to complete this task. The number of correctly transcribed symbols is used as an indicator of performance-lower scores indicate underperformance.

\section{MRI data acquisition}

The images were collected at Tianjin First Central Hospital using a 3.0 T magnetic resonance scanner (TIMTrio, Siemens Medical Solutions) and an eight-channel head coil. For functional images, blood oxygen level-dependent single-shot echoplanar sequence parallel to the anterior commissure-posterior commissure plane [repetition time, $2500 \mathrm{~ms}$; echo time, $30 \mathrm{~ms}$; flip angle, $90^{\circ}$; field of view, $220 \times 220 \mathrm{~mm}^{2}$; matrix, $96 \times 96$; iPAT (integrated parallel imaging technique) factor, 2; number of slices, 40 ; slice thickness, $3 \mathrm{~mm}$; intersection gap, $0.3 \mathrm{~mm}$; 200 volumes; acquisition time, $8.5 \mathrm{~min}$ ] was performed. During the scan, all subjects were instructed to close their eyes and remain awake, they were asked to think of nothing in particular. For structural images, a sagittal 3D T1weighted MPRAGE (magnetization-prepared rapid acquisition gradient echo) sequence was used with the following settings: repetition time, $1900 \mathrm{~ms}$; echo time, $3 \mathrm{~ms}$; inversion time, $900 \mathrm{~ms}$; flip angle, $9^{\circ}$; number of slices, 176; slice thickness, $1 \mathrm{~mm}$; matrix, $256 \times 256$. During the whole scanning period, foam pads were used to reduce head movement and ear plugs were used to reduce scanner noise. All acquired images were visually inspected and confirmed by an independent radiologist to be free of any problems occurring during image acquisition and of any significant structural lesions.

\section{Data preprocessing}

Functional imaging data were preprocessed using SPM12 software (https://www.fil.ion.ucl.ac.uk/spm/). For each participant, the first 10 volumes were removed to allow for dynamic equilibrium and adaption to the scanning circumstances. The remaining functional images were corrected for time delays between slices using slice timing. A six-parameter rigid body transformation in the realignment analysis was performed to correct head motion. Participants with a translation exceeding $3 \mathrm{~mm}$ and a rotation of $>1.5^{\circ}$ were excluded for further analyses. In the present study, no such participants were removed. Each individual subject's structural image was then segmented into gray matter, white matter (WM), and CSF for normalization after coregistration to the mean functional image. All functional images were spatially normalized to the standard MNI (Montreal Neurologic Institute) space using the generated parameters at an isotropic voxel size of $3 \mathrm{~mm}$. Finally, normalized functional images were smoothed with a $4 \mathrm{~mm}$ full-width at half-maximum Gaussian filter aiming to improve the signal-to-noise ratio. A linear detrended and temporally bandpass filtered $(0.01 \mathrm{~Hz}<f<0.08 \mathrm{~Hz})$ procedure was performed to reduce the effects of low- frequency drift and high-frequency physiological noises (Biswal et al., 1995). Several sources of spurious variance along with their temporal derivative were used to reduce the physiological noise and remove artifacts by the following linear regression: averaged signals from WM, CSF, and six head motion parameters.

\section{Whole-brain functional connectivity analysis}

To obtain the whole-brain FC matrix, a prior Anatomical Automatic Labeling template was used to divide the whole brain into 116 anatomic regions of interest (ROIs), including 78 cortical, 12 subcortical, and 26 cerebellar regions (Tzourio-Mazoyer et al., 2002). A representative time series was extracted by averaging the time series of all voxels within each ROI. Then, Pearson's correlation analyses were performed between each pair of ROls to calculate the correlation coefficients, followed by the normalization with a Fisher $z$ score transformation. A symmetric functional connectivity matrix $(116 \times 116)$ was generated for each subject. The triangular portion of the adjacency matrix was extracted and transformed to a vectoral feature space with 6670 dimensions.

\section{Network-based statistic}

To localize specific pairs of brain regions between which functional connectivity was altered in cirrhotic 
patients, we used the network-based statistic (NBS) approach (Zalesky et al., 2010). We first performed twosample one-tailed $t$ tests in an element-by-element manner on those connections that were significantly nonzero $(p<0.05$, Bonferroni corrected) in at least one participant. Then, a primary threshold $\left(p<1 \times 10^{-4}\right.$ in this study; Wang et al., 2013) was applied to define a set of suprathreshold links within which any connected components and their size (defined as the number of links included in these components) were determined. To estimate the significance for each component, a null distribution of connected component size was derived empirically using a nonparametric permutation approach (10,000 permutations). For each permutation, all subjects were reallocated randomly into two groups, and two-sample one-tailed $t$ tests were conducted for the same set of connections mentioned above. The same primary threshold $\left(p<1 \times 10^{-4}\right)$ was then used to generate suprathreshold links within which the maximal connected component size was recorded. Finally, for a connected component of size $\mathrm{M}$ (i.e. number of edges) found in the right grouping of control subjects and patients, the corrected $p$ value was determined by calculating the proportion of the 10,000 permutations for which the maximal connected component was larger than $\mathrm{M}$.

NBS was first applied to compare the FC differences between control subjects and pre-LT patients to show the disrupted connectivity induced by cirrhosis. NBS was then applied to compare control subjects and post-LT patients to show whether there was still impaired FC after the LT. Finally, NBS was conducted to compare pre-LT and post-LT patients to investigate the FC recovery and hyperconnectivity.

\section{Connectome behavior predictive mapping}

Connectome behavior predictive mapping and all statistics were assessed using MATLAB. To investigate reliable biomarkers in predicting task performance in restingstate whole-brain FC, a completely data-driven approach of CPM was used for HCs. CPM searched all possible pairs of regions and their associated connectivity values, constructing a model (the "connectome") that maximally fitted behavioral scores (Finn et al., 2015; Rosenberg et al., 2017; Shen et al., 2017) using cross-validation. Specifically, linear regressions were run between each edge in the connectivity matrix in the resting state and DST/NCT scores. The resulting $p$ value for each regression was recorded in a $116 \times 116$ symmetric matrix, amounting to 6670 different linear regression significance values. To find the most meaningful associations between specific connectivity and the task performance, the resulting $r$ values were held to a statistical threshold of $p<0.01$ and separated into positive tails (edges whose connectivity strength indexed higher performance scores across subjects) and negative tails (edges whose connectivity strength indexed lower performance scores across subjects). Positive and negative values in CPM were defined as a Pearson's correlation coefficient $r$ value in terms of their correlation with behaviors, regardless of better or worse performance. In our psychomotor cognitive tasks, a higher DST score represented better performance, whereas a higher NCT score represented worse per- formance. Therefore, a positive $r$ value in CPM for the DST score indicates stronger functional connectivity related to better cognitive performance. Whereas a negative $r$ value for the NCT score indicates stronger functional connectivity related to better performance.

A single summary statistic, network strength, was used to characterize each participant's degree of connectivity in the positive and negative tails. Positive network strength was calculated by averaging the edge strengths (Fisher-normalized $r$ values) from a participant's connectivity matrix in the edges of the positive tail, and negative network strength was calculated by averaging the $r$ values of the edges in the negative tail. Finally, network strength was used to predict performance scores across subjects using fivefold cross-validation (Rosenberg et al., 2016; Liu et al., 2021). Specifically, all control participants were randomly split into five subgroups. Among the five groups, one group was selected as the test dataset and the remaining four groups were used to train a CPM model to search for predictive functional connectivity for each of the cognitive task scores (DST, NCT). For the training, a linear regression model was used to fit the average value of obtained functional connectivity with each cognitive performance score. This trained model was then applied to predict performance scores in the test dataset. This process repeated 5 times until all 5 groups were tested. To validate the predictive accuracy and the robustness of the functional connectivity identified by CPM models, we correlated predicted performance scores with the observed performance scores for all participants. If the correlation was reported to be significant, then the consensus of functional connectivity maps obtained from all of the five CPM models was collected as the connectome predictor.

Meaningful connectivity-based neuromarkers discovered by CPM in control subjects were then applied in cirrhotic patients before and after LT as a post hoc analysis to evaluate whether the network can still predict subjects' task performance, which subsequently evaluates the recovery of brain-behavior relations.

\section{Data availability}

Anonymized data will be shared by request from any qualified investigator who provides a methodologically sound proposal, or for the purpose of replicating procedures and results presented in the present study. The code/software described in the article is freely available online at https://github.com/bigting84/Cirhosis.

\section{Results}

\section{Demographics and clinical data}

Demographics and clinical data for all subjects are summarized in Table 1. There were no meaningful differences in sex, age, or education level among OHE, non$\mathrm{OHE}$, and $\mathrm{HC}$ groups (two-way $t$ test, $p$ values $>0.05$ ). For both the non-OHE and OHE groups, liver function improved significantly (albumin, total bilirubin, blood ammonia, $p \leq 0.01)$ or showed a tendency toward restoration (prothrombin time) 1 month after LT (Table 1). 
Table 1: Demographic, neuropsychological, and biochemical information from the dataset

\begin{tabular}{|c|c|c|c|c|c|c|}
\hline & & Pre-L & $=64)$ & Post-I & $=36)$ & \\
\hline Protocols & $\begin{array}{l}\mathrm{HCs} \\
(n=32)\end{array}$ & $\begin{array}{l}\text { non-OHE } \\
(n=36)\end{array}$ & $\begin{array}{l}\mathrm{OHE} \\
(n=28)\end{array}$ & $\begin{array}{l}\text { non-OHE } \\
(n=23)\end{array}$ & $\begin{array}{l}\text { OHE } \\
(n=13)\end{array}$ & $p$ Value \\
\hline Sex & & & & & & $0.841^{a}$ \\
\hline Male & 21 & 24 & 20 & 16 & 10 & \\
\hline Female & 11 & 12 & 8 & 7 & 3 & \\
\hline Age (years) & $51.2 \pm 7.2$ & $48.1 \pm 9.9$ & $49.1 \pm 8.6$ & $47.4 \pm 9.4$ & $49.1 \pm 9.3$ & $0.272^{b}$ \\
\hline Education (years) & $12.7 \pm 2.6$ & $11.7 \pm 2.8$ & $12.4 \pm 3.0$ & $12.2 \pm 2.5$ & $12.6 \pm 3.9$ & $0.436^{b}$ \\
\hline $\mathrm{NCT}(\mathrm{s})$ & $40.4 \pm 10.2$ & $56.5 \pm 32.1$ & $59.8 \pm 22.8$ & $44.5 \pm 21.8$ & $45.0 \pm 15.6$ & $\begin{array}{l}0.009^{c} / 0.007^{d} \\
0.357^{e} / 0.253^{f}\end{array}$ \\
\hline DST (score) & $47.8 \pm 10.5$ & $39.2 \pm 13.7$ & $34.9 \pm 16.5$ & $48.3 \pm 15.0$ & $42.3 \pm 17.4$ & $\begin{array}{l}0.010^{c} / 0.006^{d} \\
0.897^{e} / 0.200^{f}\end{array}$ \\
\hline Prothrombin time (s) & & $17.8 \pm 6.3$ & $17.4 \pm 4.5$ & $14.8 \pm 7.7$ & $13.3 \pm 6.4$ & $0.105^{g} / 0.022^{h}$ \\
\hline Albumin (mg/dl) & & $33.2 \pm 8.3$ & $31.9 \pm 5.8$ & $40.5 \pm 6.0$ & $39.3 \pm 5.5$ & $0.000^{g} / 0.001^{h}$ \\
\hline Total bilirubin (mg/dl) & & $78.2 \pm 104.0$ & $64.9 \pm 54.5$ & $16.3 \pm 10.5$ & $15.1 \pm 8.1$ & $0.001^{g} / 0.000^{h}$ \\
\hline Blood ammonia ( $\mu \mathrm{mol} / \mathrm{l})$ & & $61.8 \pm 21.9$ & $84.60 \pm 29.5$ & $37.0 \pm 10.0$ & $48.2 \pm 22.4$ & $0.000^{g} / 0.000^{h}$ \\
\hline Child-Pugh score & & & & & & \\
\hline Class $A$ & & 5 & 0 & 4 & 0 & \\
\hline Class B & & 14 & 6 & 9 & 2 & \\
\hline Class C & & 17 & 22 & 10 & 11 & \\
\hline
\end{tabular}

Data are presented as the mean \pm SD.

${ }^{a}$ The $p$ value was obtained by the Pearson $\chi^{2}$ test (two-tailed).

b The $p$ value was obtained by the one-way ANOVA test among the three groups (two-tailed);

${ }^{c}$ The $p$ value was obtained by the two-sample $t$ test between pre-LT in non-OHE group and HCs (two-tailed).

${ }^{d}$ The $p$ value was obtained by the two-sample $t$ test between pre-LT in OHE group and HCs (two-tailed).

${ }^{e}$ The $p$ value was obtained by the two-sample $t$ test between post-LT in non-OHE group and HCs (two-tailed).

${ }^{f}$ The $p$ value was obtained by the two-sample $t$ test between post-LT in OHE group and HCs (two-tailed).

${ }^{g}$ The $p$ value was obtained by the two-sample $t$ test between post-LT and pre-LT in non-OHE group (two-tailed).

${ }^{\mathrm{h}}$ The $p$ value was obtained by the two-sample $t$ test between post-LT and pre-LT in OHE group (two-tailed).

\section{Behavior score comparison}

As expected, OHE and non-OHE groups before LT underperformed compared with $\mathrm{HCs}$-patients took longer to complete NCT tasks ( $p$ values $<0.009$ ) and had lower scores in DST tasks ( $p$ values <0.01). One month after LT, compared with HCs, both the OHE and non-OHE patients showed comparable performance in both DST ( $p$ values $>0.25)$ and NCT ( $p$ values $>0.20)$ tasks. The increased performance in DST scores and decreased performance in NCT scores suggests an enhancement of cognitive capability. Also, they may indicate cognitive recovery after LT for cirrhotic patients regardless of a history of OHE (Table 1).

\section{Disrupted functional connectivity in cirrhosis}

NBS was first applied to compare the FC differences between control subjects and pre-LT cirrhosis. For patients without $\mathrm{OHE}$, using the cluster-defining threshold of $p<1 \times 10^{-4}$ (explained in Materials and Methods), a single network of 17 connections among 16 brain regions was revealed, showing decreased functional connectivity in the non-OHE group ( $p=0.037$, corrected). We found that the decreased connectivity was mostly found in the connections among subcortical nuclei such as amygdala, putamen, and pallidum as well as cortical regions including orbital fontal, insular, and cingulate cortices (Fig. 2A).

For OHE patients, a single network of 65 connections linking 47 brain regions was revealed with decreased functional connectivity $(p=0.006$, corrected). The connectivity was mostly found in the connections involving the following structures: subcortical nuclei (amygdala, putamen, pallidum), limbic system (temporal cortex, cingulate cortex, parahippocampal gyrus), orbital frontal cortex, and other frontal, parietal cortex and cerebellum brain regions (Fig. 2B).

NBS was then applied to compare the FC differences between control subjects and post-LT cirrhosis. There were no significantly different connections found in both $\mathrm{OHE}$ and non-OHE patients.

\section{Reversed functional connectivity after LT}

Furthermore, using the cluster defining threshold of $p<1 \times 10^{-4}$, one additional network of 19 connections between 17 brain regions was revealed, showing increased functional connectivity in the OHE group $(p=0.009$, corrected) after LT relative to the connectivity found before LT. We found that this increased connectivity was not involved in the disrupted network seen before LT, but mostly found in the connections between cerebellum subregions, and between cerebellum and occipital cortex, orbital-frontal cortex, and limbic systems (Fig. 3A). A post hoc analysis using two-sample $t$ test revealed that the network strength of this network in OHE post-LT scans was significantly higher than healthy control subjects $(p=0.008$; Fig. $3 B)$, suggesting an LT-induced hyperconnectivity for OHE patients only.

\section{Brain-behavior model establishment in healthy control subjects: cross-group validation}

A fivefold cross-validation was applied to evaluate whether the networks selected from CPM could be generalized to predict unseen subjects. This step was critical because the CPM used was constructed using healthy 
A Disrupted network for non-OHE patients

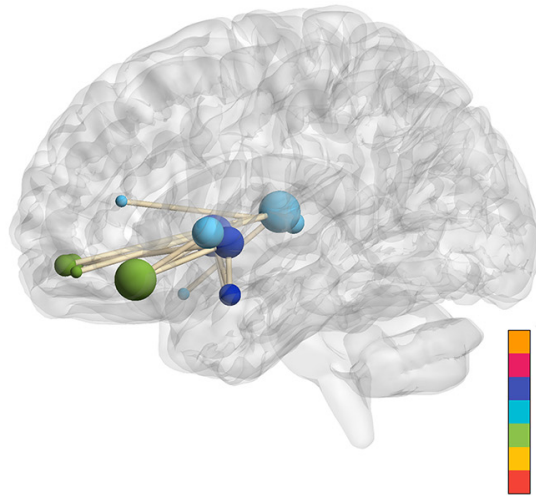

B

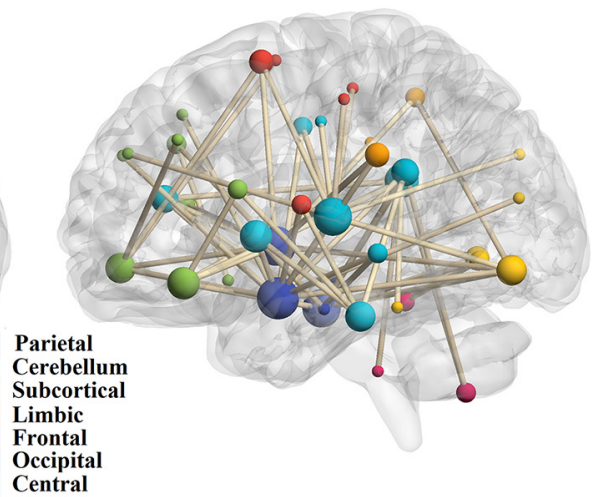

Figure 2. The disrupted resting-state functional networks for pre-LT non-OHE and OHE patients detected by NBS. $\boldsymbol{A}$, The region pairs showing significantly decreased functional connections in non-OHE patients compared with control subjects. These connections formed a single connected network with 16 nodes and 17 connections, which was significantly (NBS, $p=0.037$, corrected) abnormal in the patients. The decreased connectivity was mostly found in the connections among subcortical nuclei such as amygdala, putamen, pallidum, and orbital frontal cortex. $\boldsymbol{B}$. The region pairs showing decreased functional connections in OHE patients. These connections formed a single connected network with 47 nodes and 65 connections, which was significantly (NBS, $p=0.006$, corrected) abnormal in the patients. The connectivity was mostly found in the connections involving the following structures: subcortical nuclei (amygdala, putamen, pallidum), limbic system (temporal cortex, cingulate cortex, parahippocampal gyrus), orbital frontal cortex, and other frontal, parietal cortex and cerebellum brain regions. Functional connectivity was visualized using the BrainNetViewer toolbox in MATLAB (Xia et al., 2013), and an MNI-ICBM152 brain template (http://www.bic.mni.mcgill.ca/ ServicesAtlases/ICBM152NLin2009) was used to show the brain coordinates.

individuals. Once established, the selected functional connectivity would be used as a biomarker to evaluate the relationships between functional connectivity and behavioral outcomes before and after LT for the cirrhotic patient group. To this end, the positive and negative network models described in the Materials and Methods section were used.

In healthy control subjects, our results showed that the CPM models trained on positive networks predicted the DST task scores of unseen individuals (correlation
A

\section{Hyper-active network for OHE after LT}

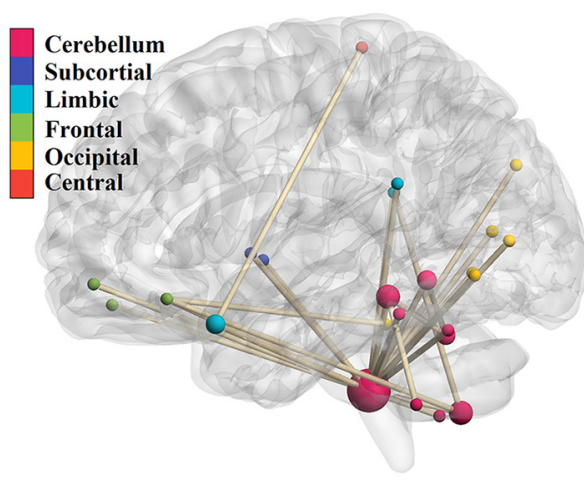

B

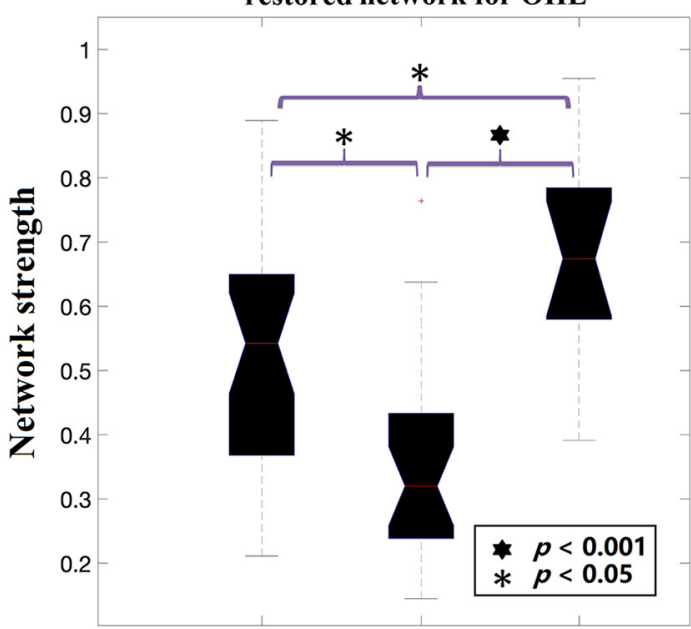

Control Pre LT Post LT

Figure 3. The restored resting-state functional network for post-LT OHE patients detected by NBS. $\boldsymbol{A}$, The region pairs showing increased functional connections in OHE patients after LT compared with pre-LT scans. These connections formed a single connected network with 19 nodes and 17 connections, which was significantly ( $p=0.009$, corrected) higher after LT. The increased connectivity was mostly found in the connections between cerebellum subregions, and between cerebellum and occipital cortex, orbital-frontal cortex, and limbic systems. B, Post hoc analysis comparing the strength of the network detected in post-LT OHE patients in three groups. The strength of the network after LT was significantly higher than that in control subjects (two-sample $t$ test, $p=0.008$, corrected), suggesting an LT-induced hyperconnectivity within the detected network. 


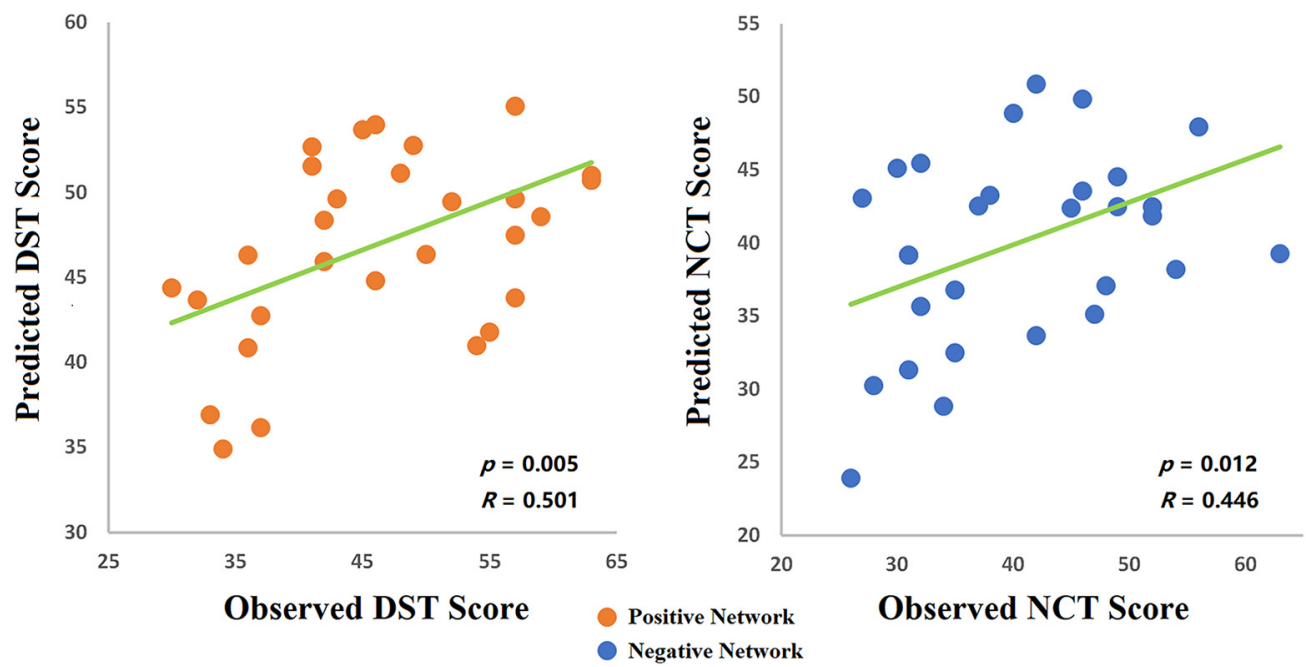

Figure 4. Cross-validation for the CPM. Functional connectivity models predicting cognitive task performance in healthy individuals. Scatter plots show correlations between observed performance scores and predictions by positive (left, for DST) and negative (right, for NCT) networks. Network models were iteratively trained on resting-state data from training subjects in the control group and tested on resting-state data from the individuals that were left out. For negative networks in the DST task and positive networks in the NCT network, no meaningful correlations were found.

between predicted and observed DST scores: $r=0.501$, $p=0.005)$, and the models trained on negative networks predicted NCT scores (correlation between predicted and observed: $r=0.446, p=0.012$; Fig. 4). Therefore, the positive network model predicting DST scores and the negative network model predicting NCT scores were further used to explore brain-behavior relationships in OHE and non-OHE patients.

Because the connections selected in each training cycle (among the five folds) were not completely identical, we extracted the connections that consistently predicted cognitive test performance in all cycles. In this manner, 6 connections from the positive network predicting DST and 12 connections from negative network predicting NCT were selected (Fig. 5B). The DST and NCT represented similar cognitive functions, and we found that their values significantly correlated with one another $(r=-0.781, p<0.0001)$. Thus, we constructed a network that combined connections that were included in both NCT negative networks and DST positive networks, and we called this network the "psychomotor network" (PMN). The PMN was then used to predict cognitive performance in patients before and after LT.

\section{Brain-behavior relationships in patients before and after LT}

For each patient, we first computed network strength by averaging the edge strengths within the PMN, and then conducted linear models relating the PMN network strength to NCT and DST scores. The PMN strength did not predict cognitive performance for pre-LT scans of either OHE or nonOHE patients ( $p$ values $>0.319$ ). However, after LT, the cognitive performance scores in non-OHE patients were predicted by PMN strength when analyzing their post-LT scans (DST: $F_{(1,21)}=7.67, p=0.011, r=0.517, \beta=12.58$; NCT: $F_{(1,21)}=4.174, p=0.049, r=-0.424, \beta=-21.33$; Fig. 5), but the scores in OHE patients were not ( $p$ values $>0.354$ ).

\section{OHE impacts the neural-behavior relationship after LT}

Previously, we found that the PMN strength significantly predicted cognitive performance only in healthy control subjects and non-OHE patients after LT. Accordingly, we further assessed whether OHE played a moderative role in the predictive relationship between resting-state connectivity and cognitive performance in post-LT scans. Moderated regression analyses assessed the association of PMN strength with DST and NCT scores, including OHE as a moderator. These analyses used unstandardized regression coefficients and 95\% bias-corrected confidence intervals (Cls) from 10,000 bootstrap estimates (Hayes, 2017, model 3). Results revealed a significant interaction between $\mathrm{OHE}$ and non-OHE groups regarding the relationship between resting-state network strength and behavioral performance $(p=0.029 ; 95 \% \mathrm{Cl}$, $1.9423,23.2147)$. No such relationships between OHE and non-OHE groups were found before LT.

Similarly, moderated mediation analyses also assessed the association of network strength with NCT scores, including $\mathrm{OHE}$ as a moderator. Results revealed a trend of the interaction effect between OHE and non-OHE groups $(p=0.061)$ in post-LT scans. No effect was found in preLT scans.

\section{Discussion}

In this study, we investigated the impairment of functional connectivity in cirrhotic patients and the recovery of impaired functional connectivity after LT by strategically using whole-brain functional connectivity comparisons and connectome-behavior predictive-mapping approaches. We found that the transplant surgery facilitated a recovery of the disrupted functional connectivity in cirrhotic patients. Furthermore, it was shown that OHE patients became abnormally hyperactive in a specific functional network after LT, which was not part of the initially disrupted network, 


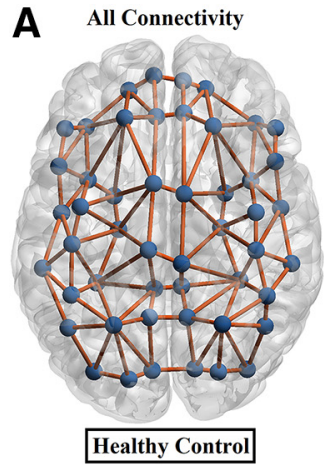

B

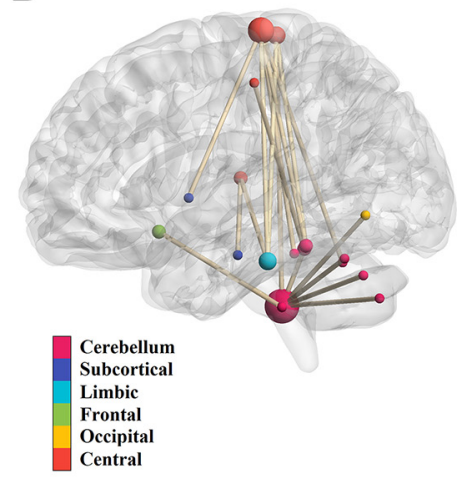

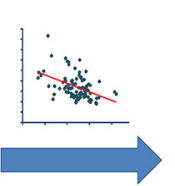

Cross Validataion

Healthy Control

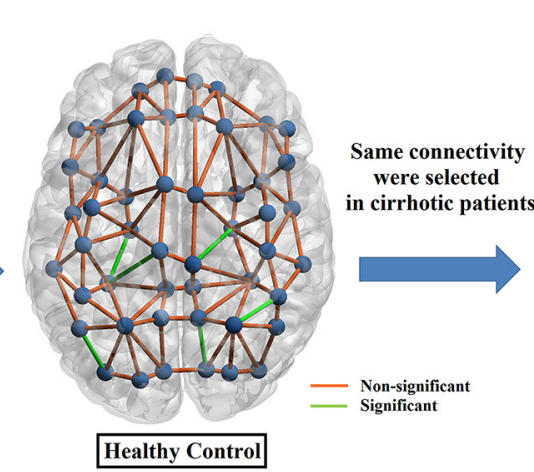

C
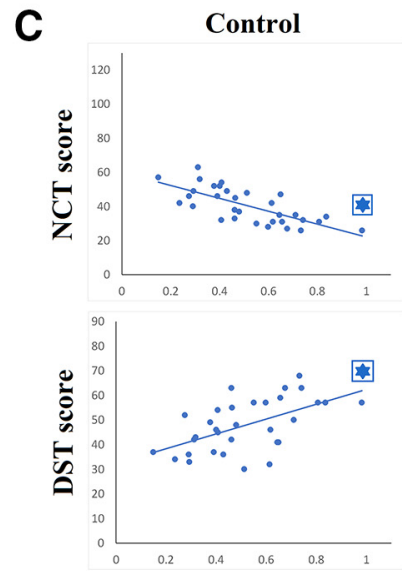

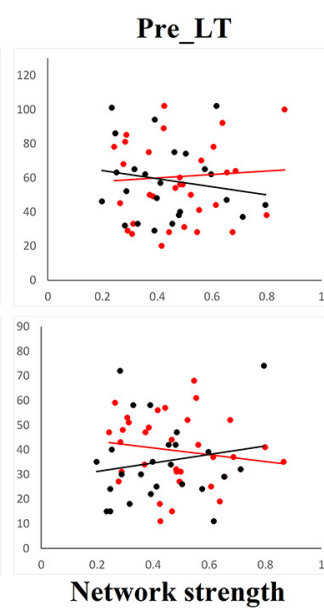

Selected Connectivity

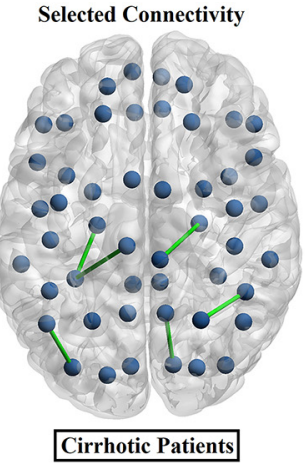

\begin{tabular}{|l|} 
PST Score \\
Predict \\
NCT Score
\end{tabular}

Cirrhotic Patients

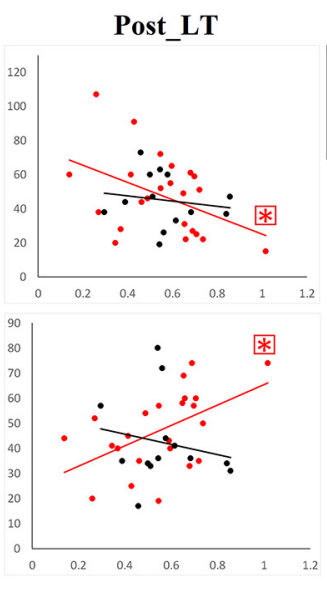

Figure 5. The connectivity-based neuromarkers obtained using connectome-behavior predictive mapping. $\boldsymbol{A}$, Connectomebehavior predictive mapping found every single connection that had a meaningful relationship $(p<0.01)$ to NCT/DST scores in HCs using cross-validation. This meaningful connectivity was then used to predict NCT/DST performance for patients before and after LT. B. The region pairs with functional connections negatively predicting NCT scores and positively predicting DST scores in control subjects. These connections formed a single connected network with 19 nodes and 18 connections, and we call this network the PMN. The predictive connectivity in PMN was mostly found in the connections linked to cerebellum subregions and paracentral cortex. C, Post hoc analysis comparing the predictive power of PMN network strength for NCT and DST scores in both pre-LT and post-LT scans. Before LT, network strength in PMN did not predict NCT/DST scores for both OHE and non-OHE patients. After LT, however, the cognitive test scores were predicted by PMN network strength when analyzing non-OHE patients only (DST: $r=0.517, p=0.011 ; \mathrm{NCT}: r=-0.424, p=0.049$ ), but not OHE patients.

suggesting a reorganization of the brain network in OHE patients in response to the surgery. Exploring functional connectivity in relation to psychomotor behavioral performance, we observed that the predictive relationship between functional connectivity and behavioral performance seen in control subjects was impaired in cirrhotic patients. This impaired relationship was recovered after LT in patients without OHE history only. The moderator role of OHE suggested that the presence of OHE may hinder normal brain connectivity recovery after LT in cirrhotic patients.

\section{Neural and cognitive recovery difference between OHE and non-OHE patients after LT}

Several interesting findings were obtained from the NBS-based comparison and post hoc analysis among groups, as follows: (1) OHE patients exhibited a larger number of, and more widely distributed, disrupted FC than non-OHE patients, suggesting more serious brain impairment by OHE; (2) no significantly decreased FC was found in post-LT scans compared with control subjects (i.e., disrupted networks for OHE and non-OHE both recovered); and (3) hyperconnectivity was found in postLT scans for OHE patients only, indicating the most significantly increased connections after LT were actually abnormal (higher than those in control subjects). Note that neither OHE nor non-OHE patients exhibited significantly disrupted connectivity after LT compared with control subjects. However, the NBS revealed the significantly increased connectivity in OHE patients only after LT, compared with before LT. It is not clear whether the recovery of the initially disrupted functional connectivity occurs partially or fully (for both OHE and non-OHE patients) because NBS is a relatively rigorous statistical approach and may not capture the connectivity difference between preoperative and postoperative scans. The enhanced functional connectivity identified in OHE patients was unlikely to be naturally recovered connectivity, as it was not part of the network that was initially disrupted in the 
patients before LT. Rather, it could be an abnormally established or reorganized network in response to LT under severe brain impairment because of the presence of OHE.

Since hyperconnectivity in response to brain injury or insult has become common in clinical brain network studies (Hillary et al., 2015; Jones et al., 2016; Kim et al., 2020), it might be inappropriate to simply assume that the hyperconnectivity after LT as a means of better recovery for OHE. Instead, hyperconnectivity might represent a reorganization of the functional network in response to the initial damage (probably irreversible) of $\mathrm{OHE}$, or another source of brain insults, as an adverse surgical effect (Filippini et al., 2009; Hillary and Grafman, 2017). In other words, OHE might induce adaptive and deformed neuroplastic changes or functional reorganization during the brain recovery process following LT. However, it remains necessary to investigate the persistence of the observed hyperconnectivity and its long-term consequences, given the chronic engagement of additional neural resources (Hillary and Grafman, 2017).

Psychomotor neurocognitive tests revealed impaired behavioral performance for cirrhotic patients compared with healthy individuals, and performance improvement after LT. Additionally, our investigation of brain-behavior predictions for cirrhotic patients sheds light on the mechanism of cognitive recovery using a generalized PMN model. For example, the PMN was not a good predictor of performance before the LT surgery for cirrhotic patients regardless of OHE. After LT, the PMN became significantly associated with cognitive performance for patients without OHE, but not for those with OHE. Given that the OHE patients also showed better behavioral performance after LT while the association between PMN and cognitive performance was impaired, it is possible that their post-LT behavioral performance was driven by the newly established hyperconnectivity or the network reorganization induced by the possibly severe and irreversible damage of OHE. Furthermore, we found that one of the key regions in PMN-cerebellum -was largely involved in the hyperconnected network for OHE after LT, suggesting that the hypercerebellar network established in OHE patients after LT may dissociate the relationship between the PMN and behavioral performance that was observed in healthy individuals. However, hyperconnectivity after LT was not observed for non-OHE patients, possibly because the brain damage because of non-OHE cirrhosis was milder. Accompanied by the recovery of functional connectivity after $\mathrm{LT}$, the functional role of PMN in relation to NCT and DST tasks could therefore be recovered, which in turn drove the behavioral performance for non-OHE patients.

\section{Functional decoding of PMN}

Psychomotor ability refers to a wide range of actions involving physical movement related to conscious cognitive processing (Ackerman and Cianciolo, 2000; Kahol et al., 2008). It measures the coordination of multiple cognitive abilities (e.g., attention, visual, executive function, with motor movement). The main hubs (degree $\geq 5$ within
PMN) found in the PMN include paracentral cortex and posterior cerebellum (cerebellum lobe X). Paracentral cortex is located on the medial surface of the cerebral hemisphere and is the medial continuation of the precentral and postcentral gyri. Its main role is motor and sensory functions related to the contralateral lower limb. The cerebellum is mainly involved in the control and coordination of motor movement through multiple mechanisms (e.g., timing, spatial evaluation, and sensory acquisition; Stoodley and Schmahmann, 2010). Thus, paracentral cortex and cerebellum in PMN are indicative of motor regulation, which is required in psychomotor tasks. In addition, we also found the following two hubs with relative high degree (degree $\geq 2$ within PMN): inferior temporal cortex and the orbitofrontal cortex. The inferior temporal cortex has been reported as an important region associated with visual functions (Woloszyn and Sheinberg, 2012), in particular, playing an important role in visualmotor coordination. The activity in the orbitofrontal cortex is associated with some higher-order cognitive functions like emotion and emotion regulation (Rolls et al., 2020), executive function (Nejati et al., 2018), reward learning (Izquierdo, 2017), and decision-making (Du et al., 2020). Particularly, a recent study also indicated that the OFC encodes decision variables and instructs sensory areas to guide adaptive behavior (Banerjee et al., 2020). In the PMN, it is possible that the OFC plays an important role in guiding adaptive behavior to achieve a cognitive task goal in an indirect and sophisticated way. Notably, recent anatomic and functional studies also demonstrated that the cerebellum is involved in a broad range of cognitive functions in addition to its historically well known association with sensorimotor control (Strick et al., 2009; King et al., 2019). For example, the cerebellum is involved in executive function (Koziol et al., 2012), attention (Osaka et al., 2004), and emotion process (Guell et al., 2018a). Particularly, the cerebellum lobe $X$, which is one of the main hubs in the PMN, has been suggested to be a nonmotor functional area in several recent studies (Guell et al., 2018b; Guell and Schmahmann, 2020). Rather, it is considered to be associated with visual working memory and visual recognition (King et al., 2019). The exact role of the cerebellum in the recovery of OHE still needs to be further addressed in future studies.

\section{Data-driven approach may find novel and robust neuromarkers}

The data-driven approach based on NBS and CPM used in this study identified novel connections that were used for group comparison and predicting cognitive test outcomes. Previously, hypothesis-driven approaches (Qi et al., 2012; García-García et al., 2018) have limited the selection of the region of interest to the brain regions that are known to be associated with the given cognitive task. Data-driven feature selection methods (Zalesky et al., 2010; Rosenberg et al., 2016) like the approach adopted in the current study, do not have such a limitation. In this regard, our approach found that the neural mechanisms for the processing of cognitive tasks in healthy subjects 
become impaired in cirrhotic patients and can be recovered after LT in the absence of OHE. This finding implies that specific, but novel brain connections might be involved in cognitive tasks of interest for patients with cirrhosis. This is more frequently encountered in cognitive tasks that are more taxing (e.g., tasks that demand multiple cognitive resources like attention, working memory, motor, and visual-spatial components, like psychomotor neurocognitive tasks).

\section{Limitations and future directions}

There are several limitations in the current study. The cerebellum plays an important role in fMRI studies for $\mathrm{OHE}$ and cirrhosis. Although the recent literature has reported that the cerebellum facilitates successful psychomotor task performance (Medina et al., 2010; Shiroma et al., 2016), the complete role of the cerebellum in the recovery of OHE still needs to be addressed further. The current investigation of the brain functional recovery process is conducted based on patients 1 month after LT. To answer whether the recovery of cognitive function, the recovery of connectivity, the observed hyperconnectivity or functional reorganization in cognitive recovery is temporary or persistent, an extended study that includes longitudinal data with a longer follow-up after LT is needed. Our data-driven method also has its own problems: it is difficult to clarify the role of the identified functional network or brain regions when their original function is not associated with the target cognitive function. Because of the modest sample size, it is possible that some of the reported effect sizes are inflated, particularly given the high-dimensional feature space; and some of the reported results may have a higher false-positive rate (Button et al., 2013). Future studies should focus on replicating the current findings with a larger sample size.

\section{References}

Ackerman PL, Cianciolo AT (2000) Cognitive, perceptual-speed, and psychomotor determinants of individual differences during skill acquisition. J Exp Psychol Appl 6:259-290.

Ahluwalia V, Wade JB, White MB, Gilles HS, Heuman DM, Fuchs $M$, Gavis EA, Fagan A, Tinsley F, Ganapathy D, Thacker LR, Sterling RK, Stravitz RT, Puri P, Sanyal AJ, Siddiqui MS, Matherly S, Luketic V, Steinberg J, Moeller FG, et al. (2016) Liver transplantation significantly improves global functioning and cerebral processing. Liver Transpl 22:1379-1390.

Bajaj JS, Wade JB, Sanyal AJ (2009) Spectrum of neurocognitive impairment in cirrhosis: implications for the assessment of hepatic encephalopathy. Hepatology 50:2014-2021.

Bajaj JS, Schubert CM, Heuman DM, Wade JB, Gibson DP, Topaz A, Saeian K, Hafeezullah M, Bell DE, Sterling RK, Stravitz RT, Luketic V, White MB, Sanyal AJ (2010) Persistence of cognitive impairment after resolution of overt hepatic encephalopathy. Gastroenterology 138:2332-2340.

Bajaj JS, Heuman DM, Wade JB, Gibson DP, Saeian K, Wegelin JA, Hafeezullah M, Bell DE, Sterling RK, Stravitz RT, Fuchs M, Luketic V, Sanyal AJ (2011) Rifaximin improves driving simulator performance in a randomized trial of patients with minimal hepatic encephalopathy. JG 140:478-487.e1.
Banerjee A, Parente G, Teutsch J, Lewis C, Voigt FF, Helmchen F (2020) Value-guided remapping of sensory cortex by lateral orbitofrontal cortex. Nature 585:245-250.

Barch DM, Burgess GC, Harms MP, Petersen SE, Schlaggar BL, Corbetta M, Glasser MF, Curtiss S, Dixit S, Feldt C, Nolan D, Bryant E, Hartley T, Footer O, Bjork JM, Poldrack R, Smith S, Johansen-Berg H, Snyder AZ, Van Essen DC (2013) Function in the human connectome: task-fMRI and individual differences in behavior. Neuroimage 80:169-189.

Bernier RA, Roy A, Venkatesan UM, Grossner EC, Brenner EK, Hillary FG (2017) Corrigendum: dedifferentiation does not account for hyperconnectivity after traumatic brain injury. Front Neurol 8:674.

Bharath RD, Munivenkatappa A, Gohel S, Panda R, Saini J, Rajeswaran J, Shukla D, Bhagavatula ID, Biswal BB (2015) Recovery of resting brain connectivity ensuing mild traumatic brain injury. Front Hum Neurosci 9:513.

Biswal B, Yetkin FZ, Haughton VM, Hyde JS (1995) Functional connectivity in the motor cortex of resting human brain using echoplanar MRI. Magn Reson Med 34:537-541.

Button KS, loannidis JP, Mokrysz C, Nosek BA, Flint J, Robinson ES, Munafò MR (2013) Power failure: why small sample size undermines the reliability of neuroscience. Nat Rev Neurosci 14:365376.

Campagna F, Montagnese S, Schiff S, Biancardi A, Mapelli D, Angeli P, Poci C, Cillo U, Merkel C, Gatta A, Amodio P (2014) Cognitive impairment and electroencephalographic alterations before and after liver transplantation: what is reversible? Liver Transpl 20:977-986.

Cárdenas A, Ginès P (2011) Acute-on-chronic liver failure: the kidneys. Curr Opin Crit Care 17:184-189.

Castellanos NP, Paúl N, Ordóñez VE, Demuynck O, Bajo R, Campo P, Bilbao A, Ortiz T, del-Pozo F, Maestú F (2010) Reorganization of functional connectivity as a correlate of cognitive recovery in acquired brain injury. Brain 133:2365-2381.

Chen H-J, Zhu XQ, Shu H, Yang M, Zhang Y, Ding J, Wang Y, Teng GJ (2012) Structural and functional cerebral impairments in cirrhotic patients with a history of overt hepatic encephalopathy. Eur J Radiol 81:2463-2469.

Chen H-J, Zhang L, Jiang L-F, Chen Q-F, Li J, Shi H-B (2016) Identifying minimal hepatic encephalopathy in cirrhotic patients by measuring spontaneous brain activity. Metab Brain Dis 31:761769.

Cheng Y, Huang L, Zhang X, Zhong J, Ji Q, Xie S, Chen L, Zuo P, Zhang LJ, Shen W (2015) Liver transplantation nearly normalizes brain spontaneous activity and cognitive function at 1 month: a resting-state functional MRI study. Metab Brain Dis 30:979-988.

Cheng Y, Huang L-X, Zhang L, Ma M, Xie S-S, Ji Q, Zhang X-D, Zhang G-Y, Zhang X-N, Ni H-Y, Shen W (2017) Longitudinal intrinsic brain activity changes in cirrhotic patients before and one month after liver transplantation. Korean J Radiol 18:370377.

Du J, Rolls ET, Cheng W, Li Y, Gong W, Qiu J, Feng J (2020) Functional connectivity of the orbitofrontal cortex, anterior cingulate cortex, and inferior frontal gyrus in humans. Cortex 123:185199.

Ferenci P, Lockwood A, Mullen K, Tarter R, Weissenborn K, Blei AT (2002) Hepatic encephalopathy-definition, nomenclature, diagnosis, and quantification: final report of the working party at the 11th World Congresses of Gastroenterology, Vienna, 1998. Hepatology 35:716-721.

Filippini N, MacIntosh BJ, Hough MG, Goodwin GM, Frisoni GB, Smith SM, Matthews PM, Beckmann CF, Mackay CE (2009) Distinct patterns of brain activity in young carriers of the APOE- $\varepsilon 4$ allele. Proc Natl Acad Sci U S A 106:7209-7214.

Finn ES, Shen X, Scheinost D, Rosenberg MD, Huang J, Chun MM, Papademetris X, Constable RT (2015) Functional connectome fingerprinting: identifying individuals using patterns of brain connectivity. Nat Neurosci 18:1664-1671. 
García-García R, Cruz-Gómez ÁJ, Urios A, Mangas-Losada A, Forn C, Escudero-García D, Kosenko E, Torregrosa I, Tosca J, GinerDurán R (2018) Learning and memory impairments in patients with minimal hepatic encephalopathy are associated with structural and functional connectivity alterations in hippocampus. Sci Rep 8:9664.

Grefkes C, Ward NS (2014) Cortical reorganization after stroke: how much and how functional? Neuroscientist 20:56-70.

Guell X, Schmahmann J (2020) Cerebellar functional anatomy: a didactic summary based on human fMRI evidence. Cerebellum 19:1-5.

Guell X, Gabrieli JD, Schmahmann JD (2018a) Triple representation of language, working memory, social and emotion processing in the cerebellum: convergent evidence from task and seed-based resting-state fMRI analyses in a single large cohort. Neuroimage 172:437-449.

Guell X, Schmahmann JD, Gabrieli JD, Ghosh SS (2018b) Functional gradients of the cerebellum. Elife $7: \mathrm{e} 36652$.

Hayes AF (2017) Introduction to mediation, moderation, and conditional process analysis: A regression-based approach. New York, NY:Guilford publications.

Hillary FG, Grafman JH (2017) Injured brains and adaptive networks: the benefits and costs of hyperconnectivity. Trends Cogn Sci 21:385-401.

Hillary FG, Roman CA, Venkatesan U, Rajtmajer SM, Bajo R, Castellanos ND (2015) Hyperconnectivity is a fundamental response to neurological disruption. Neuropsychology 29:59-75.

Izquierdo A (2017) Functional heterogeneity within rat orbitofrontal cortex in reward learning and decision making. J Neurosci 37:10529-10540.

Jaeger J (2018) Digit symbol substitution test: the case for sensitivity over specificity in neuropsychological testing. J Clin Psychopharmacol 38:513-519.

Jones DT, Knopman DS, Gunter JL, Graff-Radford J, Vemuri P, Boeve BF, Petersen RC, Weiner MW, Jack CR Jr (2016) Cascading network failure across the Alzheimer's disease spectrum. Brain 139:547-562.

Kahol K, Leyba MJ, Deka M, Deka V, Mayes S, Smith M, Ferrara JJ, Panchanathan S (2008) Effect of fatigue on psychomotor and cognitive skills. Am J Surg 195:195-204.

Kim SY, Liu M, Hong S-J, Toga AW, Barkovich AJ, Xu D, Kim H (2020) Disruption and compensation of sulcation-based covariance networks in neonatal brain growth after perinatal injury. Cereb Cortex 30:6238-6253.

King M, Hernandez-Castillo CR, Poldrack RA, Ivry RB, Diedrichsen J (2019) Functional boundaries in the human cerebellum revealed by a multi-domain task battery. Nat Neurosci 22:1371-1378.

Koziol LF, Budding DE, Chidekel D (2012) From movement to thought: executive function, embodied cognition, and the cerebellum. Cerebellum 11:505-525.

Liu M, Backer RA, Amey RC, Splan EE, Magerman A, Forbes CE (2021) Context matters: situational stress impedes functional reorganization of intrinsic brain connectivity during problem solving. Cereb Cortex 31:2111-2124.

Matarazzo JD, Herman DO (1984) Base rate data for the WAIS-R: test-retest stability and VIQ-PIQ differences. J Clin Neuropsychol 6:351-366.

Medina KL, Nagel BJ, Tapert SF (2010) Abnormal cerebellar morphometry in abstinent adolescent marijuana users. Psychiatry Res 182:152-159.

Miyake A, Friedman NP, Emerson MJ, Witzki AH, Howerter A, Wager TD (2000) The unity and diversity of executive functions and their contributions to complex "frontal lobe" tasks: a latent variable analysis. Cogn Psychol 41:49-100.

Nejati V, Salehinejad MA, Nitsche MA (2018) Interaction of the left dorsolateral prefrontal cortex (I-DLPFC) and right orbitofrontal cortex (OFC) in hot and cold executive functions: evidence from transcranial direct current stimulation (tDCS). Neuroscience 369:109123.
Osaka N, Osaka M, Kondo H, Morishita M, Fukuyama H, Shibasaki H (2004) The neural basis of executive function in working memory: an fMRI study based on individual differences. Neuroimage 21:623-631.

Poldrack RA (2010) Mapping mental function to brain structure: how can cognitive neuroimaging succeed? Perspect Psychol Sci 5:753-761.

Poldrack RA, Yarkoni T (2016) From brain maps to cognitive ontologies: informatics and the search for mental structure. Annu Rev Psychol 67:587-612.

Qi R, Zhang L, Wu S, Zhong J, Zhang Z, Zhong Y, Ni L, Zhang Z, Li K, Jiao Q, Wu X, Fan X, Liu Y, Lu G (2012) Altered resting-state brain activity at functional MR imaging during the progression of hepatic encephalopathy. Radiology 264:187-195.

Qi R, Zhang LJ, Luo S, Ke J, Kong X, Xu Q, Liu C, Lu H, Lu GM (2014) Default mode network functional connectivity: a promising biomarker for diagnosing minimal hepatic encephalopathy: CONSORT-compliant article. Medicine (Baltimore) 93: e227.

Rolls ET, Cheng W, Du J, Wei D, Qiu J, Dai D, Zhou Q, Xie P, Feng J (2020) Functional connectivity of the right inferior frontal gyrus and orbitofrontal cortex in depression. Soc Cogn Affect Neurosci 15:75-86.

Rosenberg MD, Finn ES, Scheinost D, Papademetris X, Shen X, Constable RT, Chun MM (2016) A neuromarker of sustained attention from whole-brain functional connectivity. Nat Neurosci 19:165-171.

Rosenberg MD, Finn E, Scheinost D, Constable R, Chun M (2017) Characterizing attention with predictive network models. Trends Cogn Sci 21:290-302.

Shen X, Finn ES, Scheinost D, Rosenberg MD, Chun MM, Papademetris X, Constable RT (2017) Using connectome-based predictive modeling to predict individual behavior from brain connectivity. Nat Protoc 12:506-518.

Shiroma A, Nishimura M, Nagamine H, Miyagi T, Hokama $Y$, Watanabe T, Murayama S, Tsutsui M, Tominaga D, Ishiuchi S (2016) Cerebellar contribution to pattern separation of human hippocampal memory circuits. Cerebellum 15:645-662.

Sotil EU, Gottstein J, Ayala E, Randolph C, Blei AT (2009) Impact of preoperative overt hepatic encephalopathy on neurocognitive function after liver transplantation. Liver Transpl 15:184192.

Stoodley CJ, Schmahmann JD (2010) Evidence for topographic organization in the cerebellum of motor control versus cognitive and affective processing. Cortex 46:831-844.

Strick PL, Dum RP, Fiez JA (2009) Cerebellum and nonmotor function. Annu Rev Neurosci 32:413-434.

Tzourio-Mazoyer N, Landeau B, Papathanassiou D, Crivello F, Etard O, Delcroix N, Mazoyer B, Joliot M (2002) Automated anatomical labeling of activations in SPM using a macroscopic anatomical parcellation of the MNI MRI single-subject brain. Neuroimage 15:273-289.

Umapathy S, Dhiman RK, Grover S, Duseja A, Chawla YK (2014) Persistence of cognitive impairment after resolution of overt hepatic encephalopathy. Am J Gastroenterol 109:10111019.

Wang J, Zuo X, Dai Z, Xia M, Zhao Z, Zhao X, Jia J, Han Y, He Y (2013) Disrupted functional brain connectome in individuals at risk for Alzheimer's disease. Biol Psychiatry 73:472-481.

Weissenborn K, Ennen JC, Schomerus H, Rückert N, Hecker HJ (2001) Neuropsychological characterization of hepatic encephalopathy. J Hepatol 34:768-773.

Weissenborn K, Giewekemeyer K, Heidenreich S, Bokemeyer M, Berding G, Ahl B (2005) Attention, memory, and cognitive function in hepatic encephalopathy. Metab Brain Dis 20:359-367.

Woloszyn L, Sheinberg DL (2012) Effects of long-term visual experience on responses of distinct classes of single units in inferior temporal cortex. Neuron 74:193-205. 
Woo C-W, Chang LJ, Lindquist MA, Wager TD (2017) Building better biomarkers: brain models in translational neuroimaging. Nat Neurosci 20:365-377.

Xia M, Wang J, He Y (2013) BrainNet Viewer: a network visualization tool for human brain connectomics. PloS one 8:e68910.

Yarkoni T, Poldrack RA, Nichols TE, Van Essen DC, Wager TD (2011) Large-scale automated synthesis of human functional neuroimaging data. Nat Methods 8:665-670.

Zalesky A, Fornito A, Bullmore ET (2010) Network-based statistic: identifying differences in brain networks. Neuroimage 53:11971207.

Zeng X, Zhang LY, Liu Q, Lu CH, Wei J, Shi ZW, Huang WR, Qu LH, Xu F, Wang XH, Shi PM, Tan Y, Tan W, Yuan ZL, Xia CY, Liu YL,
Xie WF (2020) Combined scores from the EncephalApp Stroop Test, number connection test B, and serial dotting test accurately identify patients with covert hepatic encephalopathy. Clin Gastroenterol Hepatol 18:1618-1625.e7.

Zhang G, Cheng Y, Liu B (2017a) Abnormalities of voxel-based whole-brain functional connectivity patterns predict the progression of hepatic encephalopathy. Brain Imaging Behav 11:784-796.

Zhang G, Cheng Y, Shen W, Liu B, Huang L, Xie S (2017b) The short-term effect of liver transplantation on the low-frequency fluctuation of brain activity in cirrhotic patients with and without overt hepatic encephalopathy. Brain Imaging Behav 11:1849-1861. 